\title{
Z3 Research Square \\ LRRC3B polymorphisms contributed to breast cancer susceptibility in Chinese Han Population
}

\author{
Tianbo Jin \\ Xizang Minzu University \\ Linna Peng \\ Xizang Minzu University \\ Shishi Xing \\ Xizang Minzu University

\section{Dandan Li} \\ Xizang Minzu University \\ Chunjuan He \\ Xizang Minzu University

\section{Xue He} \\ Xizang Minzu University \\ Li Wang ( $\square$ wangli_xzmd361@163.com ) \\ Xizang Minzu University
}

\section{Primary research}

Keywords: breast cancer, LRRC3B, polymorphisms, susceptibility, case-control

Posted Date: August 25th, 2020

DOI: https://doi.org/10.21203/rs.3.rs-61551/v1

License: (c) (i) This work is licensed under a Creative Commons Attribution 4.0 International License. Read Full License 


\section{Abstract \\ Purpose}

$\angle R R C 3 B$ gene, as a tumor suppressor gene was involved in the development and progress of breast cancer (BC). However, the effect of $\angle R R C 3 B$ polymorphisms on $\mathrm{BC}$ has rarely been reported. In the study, we aims to evaluate the relation between $\angle R R C 3 B$ variants and $\mathrm{BC}$ risk.

\section{Methods}

Among $563 \mathrm{BC}$ patients and 552 healthy controls, ten single-nucleotide polymorphisms (SNPs) in $L R R C 3 B$ were genotyped by Agena MassARRAY. Odds ratios (OR) and 95\% confidence interval (Cl) was calculate using logistic regression model.

\section{Results}

Our study demonstrated that rs1907168 polymorphism $(\mathrm{OR}=0.71, p=0.017)$ reduced the risk of $\mathrm{BC}$ in the overall. In stratified analyses by age, $\mathrm{rs} 1907168$ decreased $(\mathrm{OR}=0.53, p=0.002)$ while rs78205284 $(\mathrm{OR}=2.83, p=0.034)$ increased $\mathrm{BC}$ susceptibility among the population at age $\leq 51$ years. Clinical parameters such as tumor size, the status of PR and Ki67 were associated with $\angle R R C 3 B$ variants. Furthermore, we found that the association of 'GATT' haplotype with an increased risk for $\mathrm{BC}$. In addition, $\angle R R C 3 B$ gene was down-regulated in $\mathrm{BC}$ tumor and had a poor prognosis in $\mathrm{BC}$ in silico analysis.

\section{Conclusion}

Our study firstly found $L R R C 3 B$ SNPs contributed to the risk of $\mathrm{BC}$, suggesting $L R R C 3 B$ variants might help to predict $\mathrm{BC}$ progression.

\section{Introduction}

Breast cancer (BC) is the most commonly diagnosed malignant tumor and the primary cancer death among women globally [1]. In China, 268,600 new cases of BC were diagnosed and there were 69,500 deaths, and its morbidity and mortality rates have been increasing annually [2]. BC is a multi-factorial, polygenic disease resulting from genetic, endocrine and environmental factors [3]. Age, serum hormone levels, family history and environmental factors have been identified as potential contributors BC susceptibility [4]. Moreover, genetic factors were the major drivers in the genesis of $\mathrm{BC}[5-7]$.

Leucine-rich repeat-containing 3B (LRRC3B), also named LRP15, is one of the transmembrane protein, having an evolutionarily conserved LRR-domain [8]. LRR-containing proteins participated in protein interaction, cell adherence, DNA repair, gene recombination and so on[9]. LRRC3B protein has reported to regulate the DNA damage and repair pathways and cell cycle progression [10]. Additionally, $L R R C 3 B$ is down-regulated in in non-small-cell lung, head and neck, gastric and breast cancers [11-14], suggesting $\angle R R C 3 B$ involvement in carcinogenesis. Abnormal expression of $\angle R R C 3 B$ in $B C$ was associated with the development and prognosis of $B C$ [13]. In addition, $L R R C 3 B$ also served an important role in $B C$ recurrence and metastasis[15]. Genetic polymorphisms may affect its gene expression, thus associated with the occurrence of disease. In spite of many researchers reported the role of $L R R C 3 B$ expression in $B C$ pathogenesis, the possible effect of $\angle R R C 3 B$ polymorphisms on $\mathrm{BC}$ have not been studied. 
Here, we investigated the frequencies distribution of $\angle R R C 3 B$ polymorphisms and the potential relationship of $L R R C 3 B$ polymorphisms with BC susceptibility in the Chinese Han females.

\section{Materials And Methods Study subjects}

1115 unrelated Chinese Han female subjects (563 BC patients and 552 healthy controls) were enrolled from the Shaanxi Provincial Cancer Hospital. BC patients had newly diagnosed and were confirmed by histopathology based on the WHO $\mathrm{BC}$ classification. The blood samples of patients was collected before chemotherapy, radiotherapy, or endocrine therapy. Patients had no any other cancer or other systemic inflammatory disease. At the mean time, age-matched healthy individuals who had a checkup at the hospital were recruited as controls. The controls had no mammary tumors by recent clinical and mammographic examinations, no cancer history, no BC family history and no mammary diseases. The study was approved by the Ethics Committee of Xizang Minzu University and in accordance with the $1964 \mathrm{Helsinki}$ Declaration. Written informed consent was obtained.

\section{Data collection and DNA extraction}

Questionnaires and medical records were used to gather the demographic and clinical features. The following data were recorded: age, estrogen receptor (ER), progesterone receptor (PR), human epidermal growth factor receptor 2 (HER2) and $\mathrm{Ki}-67$ status, clinical stage, tumor size and tumor lymph node invasion. After interview, peripheral blood (5 mL) was gathered into EDTA-coated tubes from patients and healthy controls. Genomic DNA was isolated using the GoldMag genomic DNA purification kit (GoldMag Co. Ltd., Xi『an, China).

\section{Genotyping of SNPs}

Ten candidate SNPs (rs112276562, rs6790894, rs6551121, rs6551122, rs1907168, rs73150416, rs12635768, rs6551130, rs78205284 and rs6788033) in $\angle R R C 3 B$ was selected according to the 1000 Genomes Project data, with the minor allele frequency $(\mathrm{MAF}) \geq 0.05$ in Chinese Han population and a pairwise $\mathrm{r}^{2}>0.80$. LRRC3BSNPs were genotyped by MassARRAY platform (Agena Bioscience, San Diego, CA, USA) $[16,17]$. The primers were shown in Supplementary Table 1. About $10 \%$ of the samples were randomly selected and genotyped in duplicate for quality control, and the concordance were $100 \%$.

\section{In silico analysis}

Prediction of $L R R C 3 B$ SNPs possible function was performed with HaploReg v4.1 online tool. With the purpose to assess $\angle R R C 3 B$ mRNA expression in BC, GEPIA database was used. Kaplan-Meier plotter was used to evaluate the prognosis of $\angle R R C 3 B$ expression in $\mathrm{BC}$.

\section{Data analysis}

Student's t test was performed to evaluate the age distribution. Hardy-Weinberg equilibrium (HWE) was tested by a goodness-of-fit $\chi^{2}$ test in controls. The genotype frequencies of $L R R C 3 B$ SNPs between patients and controls were analyzed by $\chi^{2}$ test. Odds ratios (OR) and 95\% confidence interval $(\mathrm{Cl})$ adjusted age were calculated, and haplotype frequencies were estimated using PLINK software. Pairwise linkage disequilibrium (LD) analysis was analyzed using Haploview software (version 4.2). $p$-values $<0.05$ were considered significant.

\section{Results}


The features of all subjects were summarized in Table 1. $563 \mathrm{BC}$ patients ( $52.05 \pm 9.81$ years) and 552 healthy controls (51.88 \pm 9.85 years) participated in the present study. No significant differences of age distribution between cases and controls ( $p=0.767)$ was found. Most patients had tumor size $>2 \mathrm{~cm}(56.0 \%)$, stage I/II $(64.8 \%)$, lymph node invasion (48.8\%), positive hormone receptor status such as ER + (67.1\%), PR +(60.6\%), or HER2 + (48.5\%) and positive Ki67 $(64.8 \%)$.

Table 1

Characteristics of patients with breast cancer and controls

\begin{tabular}{|c|c|c|c|c|}
\hline \multicolumn{2}{|l|}{ Characteristics } & \multirow{2}{*}{$\begin{array}{l}\text { Cases } \\
(n=563) \\
52.05 \pm 9.81\end{array}$} & \multirow{2}{*}{$\begin{array}{l}\text { Controls } \\
(n=552) \\
51.88 \pm 9.85\end{array}$} & \multirow{2}{*}{$\begin{array}{l}p \\
0.767\end{array}$} \\
\hline Age & Mean \pm SD (years) & & & \\
\hline & $>51$ & $297(52.8 \%)$ & 295 (53.4\%) & \\
\hline & $\leq 51$ & $266(47.2 \%)$ & $257(46.6 \%)$ & \\
\hline \multirow[t]{3}{*}{ Tumor size (cm) } & $\leq 2 \mathrm{~cm}$ & $107(19.0 \%)$ & & \\
\hline & $>2 \mathrm{~cm}$ & $315(56.0 \%)$ & & \\
\hline & Missing & $141(25.0 \%)$ & & \\
\hline \multirow[t]{3}{*}{ Stage } & $\bigotimes-\bigotimes$ & $365(64.8 \%)$ & & \\
\hline & $\bigotimes-\bigotimes$ & $162(28.8 \%)$ & & \\
\hline & Missing & $36(6.4 \%)$ & & \\
\hline \multirow[t]{3}{*}{ Lymph node invasion } & No & $260(46.2 \%)$ & & \\
\hline & Yes & $275(48.8 \%)$ & & \\
\hline & Missing & $28(5.0 \%)$ & & \\
\hline \multirow[t]{3}{*}{ ER status } & Negative & $161(28.6 \%)$ & & \\
\hline & Positive & $378(67.1 \%)$ & & \\
\hline & Missing & $24(4.3 \%)$ & & \\
\hline \multirow[t]{3}{*}{ PR status } & Negative & $212(37.7 \%)$ & & \\
\hline & Positive & $341(60.6 \%)$ & & \\
\hline & Missing & $10(1.8 \%)$ & & \\
\hline \multirow[t]{3}{*}{ HER2 status } & Negative & $91(16.2 \%)$ & & \\
\hline & Positive & $273(48.5 \%)$ & & \\
\hline & Missing & 199 (35.3\%) & & \\
\hline \multirow[t]{3}{*}{ Ki67 } & Negative & $132(23.4 \%)$ & & \\
\hline & Positive & $365(64.8 \%)$ & & \\
\hline & Missing & $66(11.7 \%)$ & & \\
\hline
\end{tabular}


The MAF of the LRRC3B SNPs were more than 0.05 (Supplementary Table 2). No deviation from the HWE for the polymorphisms examined was observed in the genotype distributions of the controls $(p>0.05)$, suggesting these subjects could represent the general population. In addition, $\angle R R C 3 B$ variants were related to enhancer histone, changed motifs and GRASP QTL hits in silico analysis.

We performed allele and genetic model analyses of ten $\angle R R C 3 B$ SNPs in the BC cases and control subjects. The genotype frequencies distribution of SNPs was shown in Table 2 and Supplementary Table 3. The distributions of A and T alleles of $\angle R R C 3 B$ rs 1907168 polymorphism were $84.6 \%$ and $15.4 \%$ in controls and $87.7 \%$ and $12.3 \%$ in patients. An association between T allele and $\mathrm{BC}$ risk $(\mathrm{OR}=0.77,95 \% \mathrm{Cl}: 0.61-0.99, p=0.037)$ was found. Similarly, rs1907168 had a decreased risk of $\mathrm{BC}$ (heterozygous: $\mathrm{OR}=0.71,95 \% \mathrm{Cl}: 0.54-0.94, p=0.017$; dominant: $\mathrm{OR}=0.72,95 \% \mathrm{Cl}: 0.55-0.95, \mathrm{p}=$ 0.019 ; and log-additive: $\mathrm{OR}=0.76,95 \% \mathrm{Cl}$ : 0.59-0.97, $p=0.030$ ). However, no significant association was found for other SNPs (Supplementary Table 3).

Table 2

Relationships between $\angle R R C 3 B$ rs 1907168 polymorphism and breast cancer risk

\begin{tabular}{|c|c|c|c|c|c|}
\hline \multirow[t]{2}{*}{ Model } & \multirow[t]{2}{*}{ Genotype } & \multirow[t]{2}{*}{ Case } & \multirow[t]{2}{*}{ Control } & \multicolumn{2}{|c|}{ Adjusted by age and gender } \\
\hline & & & & OR $(95 \% \mathrm{Cl})$ & $\mathbf{p}$ \\
\hline \multirow[t]{2}{*}{ Allele } & $A$ & 987 & 929 & 1.00 & \multirow[t]{2}{*}{0.037} \\
\hline & $\mathrm{T}$ & 139 & 169 & $0.77(0.61-0.99)$ & \\
\hline \multirow[t]{3}{*}{ Genotype } & $\mathrm{AA}$ & 432 & 388 & 1.00 & \\
\hline & AT & 123 & 153 & $0.71(0.54-0.94)$ & \multirow[t]{2}{*}{0.017} \\
\hline & TT & 8 & 8 & $0.90(0.33-2.42)$ & \\
\hline \multirow[t]{2}{*}{ Dominant } & AA & 432 & 388 & 1.00 & \multirow[t]{2}{*}{0.019} \\
\hline & AT-TT & 131 & 161 & $0.72(0.55-0.95)$ & \\
\hline \multirow[t]{2}{*}{ Recessive } & AA-AT & 555 & 541 & 1.00 & \multirow[t]{2}{*}{0.963} \\
\hline & TT & 8 & 8 & $0.98(0.36-2.62)$ & \\
\hline Log-additive & - & - & - & $0.76(0.59-0.97)$ & 0.030 \\
\hline \multicolumn{6}{|c|}{ SNP, single nucleotide polymorphism; OR, odds ratio; $95 \% \mathrm{Cl}, 95 \%$ confidence interval. } \\
\hline \multicolumn{6}{|c|}{$p$ values were calculated by logistic regression analysis with adjustments for age and gende } \\
\hline \multicolumn{6}{|c|}{$p<0.05$ means the data is statistically significant. } \\
\hline
\end{tabular}

We further conducted subgroup analyses by age to examine the effects of the $\angle R R C 3 B$ SNPs on BC risk (Table 3 ). $\angle R R C 3 B$ rs 1907168 reduced the risk of $\mathrm{BC}$ among young participants (age $<51$ years) under the allele $(\mathrm{OR}=0.69,95 \% \mathrm{Cl}$ : $0.48-0.99, p=0.043)$, heterozygote ( $\mathrm{OR}=0.53,95 \% \mathrm{Cl}: 0.35-0.80, p=0.002)$, dominant ( $\mathrm{OR}=0.58,95 \% \mathrm{Cl}: 0.39-0.86, p$ $=0.008)$ and log-additive (OR $=0.68,95 \% \mathrm{Cl}: 0.47-0.98, p=0.038)$ models. In addition, rs78205284 polymorphism (TT vs. GG, OR=2.83, 95\% Cl: 1.08-7.41, $p=0.034$; and TT vs. GG-GT, OR=2.72, 95\% Cl: $1.05-7.07, p=0.040$ ) contributed to the increased $\mathrm{BC}$ risk at age $\leq 51$ years. 
Table 3

Relationships between $\angle R R C 3 B$ polymorphisms and breast cancer risk according to the stratification by age

\begin{tabular}{|c|c|c|c|c|c|c|c|c|c|c|}
\hline \multirow[t]{2}{*}{ SNP ID } & \multirow[t]{2}{*}{ Model } & \multirow[t]{2}{*}{ Genotype } & \multicolumn{4}{|l|}{$>51$} & \multicolumn{4}{|l|}{$\leq 51$} \\
\hline & & & Case & Control & $\begin{array}{l}\text { OR } \\
(95 \% \mathrm{Cl})\end{array}$ & $p$ & Case & Control & $\begin{array}{l}\text { OR } \\
(95 \% \mathrm{Cl})\end{array}$ & $p$ \\
\hline \multirow[t]{10}{*}{ rs1907168 } & \multirow[t]{2}{*}{ Allele } & $A$ & 515 & 500 & 1.00 & \multirow[t]{2}{*}{0.336} & 472 & 429 & 1.00 & \multirow[t]{2}{*}{0.043} \\
\hline & & $\mathrm{T}$ & 70 & 90 & $\begin{array}{l}0.85 \\
(0.62- \\
1.18)\end{array}$ & & 60 & 79 & $\begin{array}{l}0.69 \\
(0.48- \\
0.99)\end{array}$ & \\
\hline & \multirow[t]{3}{*}{ Genotype } & AA & 220 & 211 & 1.00 & & 212 & 177 & 1.00 & \\
\hline & & AT & 75 & 78 & $\begin{array}{l}0.91 \\
(0.63- \\
1.32)\end{array}$ & 0.609 & 48 & 75 & $\begin{array}{l}0.53 \\
(0.35- \\
0.80)\end{array}$ & 0.002 \\
\hline & & $\mathrm{TT}$ & 2 & 6 & $\begin{array}{l}0.32 \\
(0.06- \\
1.59)\end{array}$ & 0.163 & 6 & 2 & $\begin{array}{l}2.54 \\
(0.51- \\
12.77)\end{array}$ & 0.257 \\
\hline & \multirow[t]{2}{*}{ Dominant } & $A A$ & 220 & 211 & 1.00 & \multirow[t]{2}{*}{0.437} & 212 & 177 & 1.00 & \multirow[t]{2}{*}{0.008} \\
\hline & & AT-TT & 77 & 84 & $\begin{array}{l}0.87 \\
(0.60- \\
1.25)\end{array}$ & & 54 & 77 & $\begin{array}{l}0.58 \\
(0.39- \\
0.86)\end{array}$ & \\
\hline & \multirow[t]{2}{*}{ Recessive } & AA-AT & 295 & 289 & 1.00 & \multirow[t]{2}{*}{0.172} & 260 & 252 & 1.00 & \multirow[t]{2}{*}{0.188} \\
\hline & & TT & 2 & 6 & $\begin{array}{l}0.33 \\
(0.07- \\
1.63)\end{array}$ & & 6 & 2 & $\begin{array}{l}2.95 \\
(0.59- \\
14.77)\end{array}$ & \\
\hline & $\begin{array}{l}\text { Log- } \\
\text { additive }\end{array}$ & - & - & - & $\begin{array}{l}0.83 \\
(0.59- \\
1.17)\end{array}$ & 0.287 & - & - & $\begin{array}{l}0.68 \\
(0.47- \\
0.98)\end{array}$ & 0.038 \\
\hline \multirow[t]{7}{*}{ rs78205284 } & \multirow[t]{2}{*}{ Allele } & G & 482 & 477 & 1.00 & \multirow[t]{2}{*}{0.993} & 419 & 428 & 1.00 & \multirow[t]{2}{*}{0.063} \\
\hline & & $\mathrm{T}$ & 110 & 109 & $\begin{array}{l}1.00 \\
(0.74- \\
1.34)\end{array}$ & & 113 & 86 & $\begin{array}{l}1.34 \\
(0.98- \\
1.83)\end{array}$ & \\
\hline & \multirow[t]{3}{*}{ Genotype } & GG & 201 & 197 & 1.00 & & 169 & 177 & 1.00 & \\
\hline & & GT & 80 & 83 & $\begin{array}{l}0.95 \\
(0.66- \\
1.37)\end{array}$ & 0.790 & 81 & 74 & $\begin{array}{l}1.14 \\
(0.78- \\
1.66)\end{array}$ & 0.505 \\
\hline & & $\mathrm{TT}$ & 15 & 13 & $\begin{array}{l}1.15 \\
(0.53- \\
2.48)\end{array}$ & 0.725 & 16 & 6 & $\begin{array}{l}2.83 \\
(1.08- \\
7.41)\end{array}$ & 0.034 \\
\hline & \multirow[t]{2}{*}{ Dominant } & GG & 201 & 197 & 1.00 & \multirow[t]{2}{*}{0.900} & 169 & 177 & 1.00 & \multirow[t]{2}{*}{0.207} \\
\hline & & GT-TT & 95 & 96 & $\begin{array}{l}0.98 \\
(0.69- \\
1.38)\end{array}$ & & 97 & 80 & $\begin{array}{l}1.26 \\
(0.88- \\
1.82)\end{array}$ & \\
\hline SNP, single nu & cleotide poly & norphism; O & , odds & tio; 95\% & , $95 \%$ con & dence in & erval. & & & \\
\hline
\end{tabular}




\begin{tabular}{|c|c|c|c|c|c|c|c|c|c|}
\hline \multirow[t]{2}{*}{ Recessive } & GG-GT & 281 & 280 & 1.00 & \multirow[t]{2}{*}{0.694} & 250 & 251 & 1.00 & \multirow[t]{2}{*}{0.040} \\
\hline & TT & 15 & 13 & $\begin{array}{l}1.17 \\
(0.54- \\
2,50)\end{array}$ & & 16 & 6 & $\begin{array}{l}2.72 \\
(1.05- \\
7.07)\end{array}$ & \\
\hline $\begin{array}{l}\text { Log- } \\
\text { additive }\end{array}$ & - & - & - & $\begin{array}{l}1.01 \\
(0.76- \\
1.33)\end{array}$ & 0.966 & & & $\begin{array}{l}1.33 \\
(0.98- \\
1.80)\end{array}$ & 0.071 \\
\hline \multicolumn{10}{|c|}{ SNP, single nucleotide polymorphism; OR, odds ratio; $95 \% \mathrm{Cl}, 95 \%$ confidence interval. } \\
\hline \multicolumn{10}{|c|}{$p$ values were calculated by logistic regression analysis with adjustments for age. } \\
\hline \multicolumn{10}{|c|}{$p<0.05$ indicates statistical significance. } \\
\hline
\end{tabular}

We also investigated the potential associations of $\angle R R C 3 B$ polymorphisms with clinicopathological characteristics of $\mathrm{BC}$, including tumor size, lymph node metastasis, clinical stage, the statuses of ER, PR, Her-2, and Ki67 (Table 4). We found that rs6551122 ( $\mathrm{OR}=0.51, p=0.028$ ) and rs12635768 (homozygote, $\mathrm{OR}=0.36, p=0.023$; recessive, $\mathrm{OR}=0.39, p=$ 0.032 ; log-additive, $\mathrm{OR}=0.69, p=0.043)$ polymorphisms were related to a smaller $\mathrm{BC}$ tumor size $(<2 \mathrm{~cm})$. In addition, rs112276562, rs6551122 and rs73150416 variants contributed to a lower incidence of PR-positive breast cancer (for rs112276562, heterozygote $\mathrm{OR}=0.56, p=0.002$ and dominant $\mathrm{OR}=0.63, p=0.011$; for rs6551122, heterozygote $\mathrm{OR}=$ $0.63, p=0.016$ and dominant $\mathrm{OR}=0.64, p=0.018$; and for rs 73150416 , heterozygote $\mathrm{OR}=0.57, p=0.005$, dominant $\mathrm{OR}=$ $0.61, p=0.008$, log-additive $\mathrm{OR}=0.72, p=0.035$, and allele $\mathrm{OR}=0.71, p=0.029)$. Moreover, rs6788033 was associated with a lower the expression level of Ki-67in dominant $(\mathrm{OR}=0.64, p=0.030)$, log-additive $(\mathrm{OR}=0.68, p=0.024)$ and allele $(\mathrm{OR}=0.69, p=0.025)$ models. There was no significant association of $\angle R R C 3 B$ polymorphisms with respect to the other variables like lymph node metastasis, clinical stage and ER/HER2 status. 
Table 4

The association between $\angle R R C 3 B$ polymorphisms and clinical features of breast cancer

\begin{tabular}{|c|c|c|c|c|c|c|c|c|}
\hline \multirow[t]{2}{*}{ SNPs ID } & \multirow[t]{2}{*}{ Variables } & & \multicolumn{6}{|l|}{ OR (95\%), $p$} \\
\hline & & & Homozygote & Heterozygote & Dominant & Recessive & $\begin{array}{l}\text { Log- } \\
\text { additive }\end{array}$ & Allele \\
\hline \multirow[t]{7}{*}{ rs112276562 } & $\begin{array}{l}\text { Tumour } \\
\text { size }\end{array}$ & $\begin{array}{l}< \\
2 \mathrm{~cm} / \\
\geq \\
2 \mathrm{~cm}\end{array}$ & $\begin{array}{l}0.90(0.31- \\
2.64), 0.847\end{array}$ & $\begin{array}{l}1.39(0.83- \\
2.34), 0.212\end{array}$ & $\begin{array}{l}1.31 \\
(0.80- \\
2.13) \\
0.281\end{array}$ & $\begin{array}{l}0.82 \\
(0.28- \\
2.40) \\
0.721\end{array}$ & $\begin{array}{l}1.17 \\
(0.78- \\
1.76) \\
0.441\end{array}$ & $\begin{array}{l}1.16 \\
(0.77- \\
1.75), \\
0.486\end{array}$ \\
\hline & LNM & $\begin{array}{l}(-) / \\
(+)\end{array}$ & $\begin{array}{l}0.61(0.25- \\
1.47), 0.272\end{array}$ & $\begin{array}{l}0.81(0.56- \\
1.18), 0.271\end{array}$ & $\begin{array}{l}0.78 \\
(0.55- \\
1.12) \\
0.183\end{array}$ & $\begin{array}{l}0.65 \\
(0.27- \\
1.56) \\
0.337\end{array}$ & $\begin{array}{l}0.80 \\
(0.59- \\
1.08) \\
0.145\end{array}$ & $\begin{array}{l}0.80 \\
(0.59- \\
1.08), \\
0.145\end{array}$ \\
\hline & Stage & $\nabla-\nabla / \square-\square$ & $\begin{array}{l}0.75(0.29- \\
1.96), 0.560\end{array}$ & $\begin{array}{l}0.81(0.54- \\
1.23), 0.326\end{array}$ & $\begin{array}{l}0.80 \\
(0.54- \\
1.20) \\
0.282\end{array}$ & $\begin{array}{l}0.80 \\
(0.31- \\
2.07) \\
0.648\end{array}$ & $\begin{array}{l}0.83 \\
(0.60- \\
1.17) \\
0.289\end{array}$ & $\begin{array}{l}0.84 \\
(0.60- \\
1.18) \\
0.310\end{array}$ \\
\hline & ER & $\left(\begin{array}{l}(-) / \\
+)\end{array}\right.$ & $\begin{array}{l}1.78(0.59- \\
5.40), 0.308\end{array}$ & $\begin{array}{l}0.71(0.48- \\
1.06), 0.090\end{array}$ & $\begin{array}{l}0.78 \\
(0.53- \\
1.14) \\
0.200\end{array}$ & $\begin{array}{l}2.00 \\
(0.66- \\
6.00) \\
0.218\end{array}$ & $\begin{array}{l}0.90 \\
(0.65- \\
1.24) \\
0.524\end{array}$ & $\begin{array}{l}0.90 \\
(0.65- \\
1.24) \\
0.517\end{array}$ \\
\hline & PR & $\left(\begin{array}{l}(-) / \\
+)\end{array}\right.$ & $\begin{array}{l}1.54(0.59- \\
4.03), 0.375\end{array}$ & $\begin{array}{l}0.56(0.39- \\
0.81), 0.002\end{array}$ & $\begin{array}{l}0.63 \\
(0.44- \\
0.90) \\
0.011\end{array}$ & $\begin{array}{l}1.87 \\
(0.72- \\
4.84) \\
0.196\end{array}$ & $\begin{array}{l}0.77 \\
(0.57- \\
1.04) \\
0.093\end{array}$ & $\begin{array}{l}0.77 \\
(0.57- \\
1.04), \\
0.085\end{array}$ \\
\hline & HER2 & $\left(\begin{array}{l}(-) / \\
+)\end{array}\right.$ & $\begin{array}{l}0.88(0.27- \\
2.91), 0.835\end{array}$ & $\begin{array}{l}1.30(0.76- \\
2.22), 0.339\end{array}$ & $\begin{array}{l}1.24 \\
(0.74- \\
2.06) \\
0.410\end{array}$ & $\begin{array}{l}0.81 \\
(0.25- \\
2.66) \\
0.732\end{array}$ & $\begin{array}{l}1.14 \\
(0.74- \\
1.76) \\
0.561\end{array}$ & $\begin{array}{l}1.11 \\
(0.72- \\
1.72), \\
0.626\end{array}$ \\
\hline & Ki-67 & $\begin{array}{l}< \\
10 \% / \\
\geq \\
10 \%\end{array}$ & $\begin{array}{l}1.17(0.41- \\
3.34), 0.768\end{array}$ & $\begin{array}{l}1.06(0.69- \\
1.64), 0.790\end{array}$ & $\begin{array}{l}1.07 \\
(0.70- \\
1.63) \\
0.743\end{array}$ & $\begin{array}{l}1.15 \\
(0.41- \\
3.24) \\
0.794\end{array}$ & $\begin{array}{l}1.07 \\
(0.75- \\
1.53) \\
0.713\end{array}$ & $\begin{array}{l}1.06 \\
(0.74- \\
1.51) \\
0.761\end{array}$ \\
\hline \multirow[t]{3}{*}{ rs6551122 } & $\begin{array}{l}\text { Tumour } \\
\text { size }\end{array}$ & $\begin{array}{l}< \\
2 \mathrm{~cm} / \\
\geq \\
2 \mathrm{~cm}\end{array}$ & $\begin{array}{l}0.59(0.30- \\
1.13), 0.109\end{array}$ & $\begin{array}{l}1.32(0.81- \\
2.14), 0.268\end{array}$ & $\begin{array}{l}1.08 \\
(0.69- \\
1.69) \\
0.734\end{array}$ & $\begin{array}{l}0.51 \\
(0.28- \\
0.93) \\
0.028\end{array}$ & $\begin{array}{l}0.87 \\
(0.63- \\
1.20) \\
0.393\end{array}$ & $\begin{array}{l}0.88 \\
(0.64- \\
1.21) \\
0.420\end{array}$ \\
\hline & LNM & $\left(\begin{array}{l}(-) / \\
+)\end{array}\right.$ & $\begin{array}{l}0.76(0.44- \\
1.30), 0.312\end{array}$ & $\begin{array}{l}0.92(0.64- \\
1.33), 0.670\end{array}$ & $\begin{array}{l}0.88 \\
(0.62- \\
1.26) \\
0.492\end{array}$ & $\begin{array}{l}0.79 \\
(0.48- \\
1.31) \\
0.359\end{array}$ & $\begin{array}{l}0.88 \\
(0.69- \\
1.14) \\
0.337\end{array}$ & $\begin{array}{l}0.89 \\
(0.70- \\
1.14), \\
0.367\end{array}$ \\
\hline & Stage & $\nabla-\nabla / \otimes-\square$ & $\begin{array}{l}0.66(0.36- \\
1.23), 0.194\end{array}$ & $\begin{array}{l}1.06(0.71- \\
1.57), 0.794\end{array}$ & $\begin{array}{l}0.96 \\
(0.65- \\
1.40) \\
0.825\end{array}$ & $\begin{array}{l}0.64 \\
(0.36- \\
1.15) \\
0.135\end{array}$ & $\begin{array}{l}0.88 \\
(0.67- \\
1.16) \\
0.355\end{array}$ & $\begin{array}{l}0.89 \\
(0.68- \\
1.16) \\
0.389\end{array}$ \\
\hline
\end{tabular}

SNP, single nucleotide polymorphism; OR, odds ratio; $95 \% \mathrm{Cl}, 95 \%$ confidence interval; ER, estrogen receptor; RP, progesterone receptor; HER2, human epidermal growth factor receptor 2; LNM, lymph node metastasis.

$p$ values were calculated by logistic regression analysis with adjustments for age.

$p<0.05$ indicates statistical significance. 


\begin{tabular}{|c|c|c|c|c|c|c|c|c|}
\hline & ER & $\left(\begin{array}{l}(-) / \\
+)\end{array}\right.$ & $\begin{array}{l}1.05(0.58- \\
1.92), 0.866\end{array}$ & $\begin{array}{l}0.84(0.56- \\
1.25), 0.380\end{array}$ & $\begin{array}{l}0.88 \\
(0.60- \\
1.29) \\
0.504\end{array}$ & $\begin{array}{l}1.17 \\
(0.67- \\
2.03) \\
0.582\end{array}$ & $\begin{array}{l}0.97 \\
(0.74- \\
1.28), \\
0.842\end{array}$ & $\begin{array}{l}0.98 \\
(0.75- \\
1.28), \\
0.866\end{array}$ \\
\hline & PR & $\left(\begin{array}{l}(-) / \\
+)\end{array}\right.$ & $\begin{array}{l}0.71(0.41- \\
1.23), 0.218\end{array}$ & $\begin{array}{l}0.63(0.43- \\
0.92), 0.016\end{array}$ & $\begin{array}{l}0.64 \\
(0.45- \\
0.93) \\
0.018\end{array}$ & $\begin{array}{l}0.93 \\
(0.57- \\
1.53) \\
0.776\end{array}$ & $\begin{array}{l}0.79 \\
(0.61- \\
1.02) \\
0.064\end{array}$ & $\begin{array}{l}0.80 \\
(0.62- \\
1.03), \\
0.078\end{array}$ \\
\hline & HER2 & $\begin{array}{l}(-) / \\
(+)\end{array}$ & $\begin{array}{l}0.96(0.42- \\
2.17), 0.918\end{array}$ & $\begin{array}{l}0.83(0.49- \\
1.39), 0.475\end{array}$ & $\begin{array}{l}0.85 \\
(0.52- \\
1.40) \\
0.524\end{array}$ & $\begin{array}{l}1.07 \\
(0.50- \\
2.28) \\
0.860\end{array}$ & $\begin{array}{l}0.93 \\
(0.65- \\
1.34) \\
0.699\end{array}$ & $\begin{array}{l}0.93 \\
(0.66- \\
1.32), \\
0.690\end{array}$ \\
\hline & Ki-67 & $\begin{array}{l}< \\
10 \% / \\
\geq \\
10 \%\end{array}$ & $\begin{array}{l}1.32(0.69- \\
2.52), 0.408\end{array}$ & $\begin{array}{l}1.38(0.90- \\
2.13), 0.140\end{array}$ & $\begin{array}{l}1.37 \\
(0.91- \\
2.06) \\
0.133\end{array}$ & $\begin{array}{l}1.10 \\
(0.60- \\
2.02) \\
0.757\end{array}$ & $\begin{array}{l}1.21 \\
(0.90- \\
1.65) \\
0.212\end{array}$ & $\begin{array}{l}1.21 \\
(0.90- \\
1.63), \\
0.203\end{array}$ \\
\hline rs73150416 & $\begin{array}{l}\text { Tumour } \\
\text { size }\end{array}$ & $\begin{array}{l}< \\
2 \mathrm{~cm} / \\
\geq \\
2 \mathrm{~cm}\end{array}$ & $\begin{array}{l}0.71(0.26- \\
1.95), 0.505\end{array}$ & $\begin{array}{l}1.22(0.71- \\
2.07), 0.473\end{array}$ & $\begin{array}{l}1.11 \\
(0.68- \\
1.82), \\
0.676\end{array}$ & $\begin{array}{l}0.67 \\
(0.25- \\
1.85) \\
0.444\end{array}$ & $\begin{array}{l}1.01 \\
(0.68- \\
1.51) \\
0.953\end{array}$ & $\begin{array}{l}0.99 \\
(0.66- \\
1.50), \\
0.972\end{array}$ \\
\hline & LNM & $\left(\begin{array}{l}(-) / \\
+)\end{array}\right.$ & $\begin{array}{l}0.54(0.22- \\
1.34), 0.183\end{array}$ & $\begin{array}{l}0.79(0.54- \\
1.16), 0.226\end{array}$ & $\begin{array}{l}0.75 \\
(0.52- \\
1.09) \\
0.130\end{array}$ & $\begin{array}{l}0.58 \\
(0.24- \\
1.42) \\
0.232\end{array}$ & $\begin{array}{l}0.77 \\
(0.56- \\
1.04), \\
0.091\end{array}$ & $\begin{array}{l}0.76 \\
(0.55- \\
1.04) \\
0.087\end{array}$ \\
\hline & Stage & $\mathrm{Q}-\mathrm{Q} / \mathrm{Q}-\square$ & $\begin{array}{l}0.47(0.16- \\
1.43), 0.183\end{array}$ & $\begin{array}{l}0.84(0.55- \\
1.28), 0.405\end{array}$ & $\begin{array}{l}0.78 \\
(0.52- \\
1.18), \\
0.236\end{array}$ & $\begin{array}{l}0.50 \\
(0.16- \\
1.49) \\
0.211\end{array}$ & $\begin{array}{l}0.78 \\
(0.55- \\
1.10) \\
0.151\end{array}$ & $\begin{array}{l}0.78 \\
(0.54- \\
1.11), \\
0.159\end{array}$ \\
\hline & ER & $\left(\begin{array}{l}(-) / \\
+)\end{array}\right.$ & $\begin{array}{l}1.29(0.46- \\
3.60), 0.634\end{array}$ & $\begin{array}{l}0.75(0.50- \\
1.13), 0.169\end{array}$ & $\begin{array}{l}0.80 \\
(0.54- \\
1.18) \\
0.261\end{array}$ & $\begin{array}{l}1.40 \\
(0.50- \\
3.89) \\
0.519\end{array}$ & $\begin{array}{l}0.89 \\
(0.64- \\
1.23) \\
0.474\end{array}$ & $\begin{array}{l}0.88 \\
(0.63- \\
1.24) \\
0.464\end{array}$ \\
\hline & PR & $\left(\begin{array}{l}(-) / \\
+)\end{array}\right.$ & $\begin{array}{l}0.95(0.39- \\
2.34), 0.916\end{array}$ & $\begin{array}{l}0.57(0.39- \\
0.84), 0.005\end{array}$ & $\begin{array}{l}0.61 \\
(0.42- \\
0.88), \\
0.008\end{array}$ & $\begin{array}{l}1.13 \\
(0.46- \\
2.74), \\
0.792\end{array}$ & $\begin{array}{l}0.72 \\
(0.53- \\
0.98), \\
0.035\end{array}$ & $\begin{array}{l}0.71 \\
(0.52- \\
0.97) \\
0.029\end{array}$ \\
\hline & HER2 & $\begin{array}{l}(-) / \\
(+)\end{array}$ & $\begin{array}{l}1.26(0.34- \\
4.66), 0.730\end{array}$ & $\begin{array}{l}1.11(0.65- \\
1.89), 0.704\end{array}$ & $\begin{array}{l}1.13 \\
(0.67- \\
1.88), \\
0.652\end{array}$ & $\begin{array}{l}1.22 \\
(0.33- \\
4.48) \\
0.764\end{array}$ & $\begin{array}{l}1.11 \\
(0.72- \\
1.72) \\
0.628\end{array}$ & $\begin{array}{l}1.09 \\
(0.70- \\
1.69), \\
0.700\end{array}$ \\
\hline & Ki-67 & $\begin{array}{l}< \\
10 \% / \\
\geq \\
10 \%\end{array}$ & $\begin{array}{l}1.01(0.38- \\
2.67), 0.987\end{array}$ & $\begin{array}{l}1.03(0.66- \\
1.62), 0.895\end{array}$ & $\begin{array}{l}1.03 \\
(0.67- \\
1.58) \\
0.900\end{array}$ & $\begin{array}{l}1.00 \\
(0.38- \\
2.62) \\
0.999\end{array}$ & $\begin{array}{l}1.02 \\
(0.72- \\
1.45) \\
0.918\end{array}$ & $\begin{array}{l}1.01 \\
(0.70- \\
1.45), \\
0.966\end{array}$ \\
\hline rs12635768 & $\begin{array}{l}\text { Tumour } \\
\text { size }\end{array}$ & $\begin{array}{l}< \\
2 \mathrm{~cm} / \\
\geq \\
2 \mathrm{~cm}\end{array}$ & $\begin{array}{l}0.36(0.15- \\
0.87), 0.023\end{array}$ & $\begin{array}{l}0.81(0.50- \\
1.30), 0.378\end{array}$ & $\begin{array}{l}0.72 \\
(0.46- \\
1.12) \\
0.144\end{array}$ & $\begin{array}{l}0.39 \\
(0.16- \\
0.92), \\
0.032\end{array}$ & $\begin{array}{l}0.69 \\
(0.48- \\
0.99), \\
0.043\end{array}$ & $\begin{array}{l}0.71 \\
(0.50- \\
1.01) \\
0.058\end{array}$ \\
\hline
\end{tabular}

SNP, single nucleotide polymorphism; OR, odds ratio; $95 \% \mathrm{Cl}, 95 \%$ confidence interval; ER, estrogen receptor; RP, progesterone receptor; HER2, human epidermal growth factor receptor 2; LNM, lymph node metastasis.

$p$ values were calculated by logistic regression analysis with adjustments for age.

$p<0.05$ indicates statistical significance. 


\begin{tabular}{|c|c|c|c|c|c|c|c|c|}
\hline & LNM & $\left(\begin{array}{l}(-) / \\
+\end{array}\right.$ & $\begin{array}{l}1.27(0.59- \\
2.71), 0.541\end{array}$ & $\begin{array}{l}1.11(0.77- \\
1.59), 0.577\end{array}$ & $\begin{array}{l}1.13 \\
(0.8- \\
1.59) \\
0.495\end{array}$ & $\begin{array}{l}1.22 \\
(0.58- \\
2.57) \\
0.605\end{array}$ & $\begin{array}{l}1.12 \\
(0.84- \\
1.48), \\
0.448\end{array}$ & $\begin{array}{l}1.12 \\
(0.84- \\
1.48) \\
0.434\end{array}$ \\
\hline & Stage & $\nabla-\nabla / \nabla-\square$ & $\begin{array}{l}1.32(0.58- \\
2.98), 0.506\end{array}$ & $\begin{array}{l}1.16(0.79- \\
1.72), 0.454\end{array}$ & $\begin{array}{l}1.18 \\
(0.81- \\
1.72) \\
0.386\end{array}$ & $\begin{array}{l}1.24 \\
(0.56- \\
2.76) \\
0.594\end{array}$ & $\begin{array}{l}1.16 \\
(0.85- \\
1.57) \\
0.359\end{array}$ & $\begin{array}{l}1.15 \\
(0.85- \\
1.56) \\
0.364\end{array}$ \\
\hline & ER & $\begin{array}{l}(-) / \\
(+)\end{array}$ & $\begin{array}{l}0.88(0.38- \\
2.01), 0.759\end{array}$ & $\begin{array}{l}0.92(0.62- \\
1.35), 0.660\end{array}$ & $\begin{array}{l}0.91 \\
(0.63- \\
1.33) \\
0.627\end{array}$ & $\begin{array}{l}0.91 \\
(0.40- \\
2.05) \\
0.818\end{array}$ & $\begin{array}{l}0.93 \\
(0.68- \\
1.26), \\
0.625\end{array}$ & $\begin{array}{l}0.93 \\
(0.69- \\
1.27) \\
0.658\end{array}$ \\
\hline & PR & $\begin{array}{l}(-) / \\
(+)\end{array}$ & $\begin{array}{l}0.96(0.44- \\
2.09), 0.910\end{array}$ & $\begin{array}{l}0.87(0.60- \\
1.25), 0.448\end{array}$ & $\begin{array}{l}0.88 \\
(0.62- \\
1.25) \\
0.470\end{array}$ & $\begin{array}{l}1.01 \\
(0.47- \\
2.18) \\
0.981\end{array}$ & $\begin{array}{l}0.92 \\
(0.69- \\
1.22), \\
0.557\end{array}$ & $\begin{array}{l}0.93 \\
(0.70- \\
1.24), \\
0.628\end{array}$ \\
\hline & HER2 & $\left(\begin{array}{l}-) / \\
(+)\end{array}\right.$ & $\begin{array}{l}1.11(0.35- \\
3.51), 0.857\end{array}$ & $\begin{array}{l}0.71(0.43- \\
1.16), 0.173\end{array}$ & $\begin{array}{l}0.75 \\
(0.46- \\
1.21) \\
0.233\end{array}$ & $\begin{array}{l}1.27 \\
(0.41- \\
3.95) \\
0.675\end{array}$ & $\begin{array}{l}0.85 \\
(0.57- \\
1.26) \\
0.407\end{array}$ & $\begin{array}{l}0.86 \\
(0.58- \\
1.27) \\
0.447\end{array}$ \\
\hline & Ki-67 & $\begin{array}{l}< \\
10 \% / \\
\geq \\
10 \%\end{array}$ & $\begin{array}{l}1.80(0.59- \\
5.44), 0.300\end{array}$ & $\begin{array}{l}1.06(0.69- \\
1.62), 0.786\end{array}$ & $\begin{array}{l}1.12 \\
(0.74- \\
1.69), \\
0.589\end{array}$ & $\begin{array}{l}1.76 \\
(0.59- \\
5.26) \\
0.314\end{array}$ & $\begin{array}{l}1.16 \\
(0.82- \\
1.64), \\
0.411\end{array}$ & $\begin{array}{l}1.18 \\
(0.84- \\
1.67), \\
0.345\end{array}$ \\
\hline rs6788033 & $\begin{array}{l}\text { Tumour } \\
\text { size }\end{array}$ & $\begin{array}{l}< \\
2 \mathrm{~cm} / \\
\geq \\
2 \mathrm{~cm}\end{array}$ & $\begin{array}{l}1.12(0.43- \\
2.96), 0.813\end{array}$ & $\begin{array}{l}1.29(0.81- \\
2.07), 0.282\end{array}$ & $\begin{array}{l}1.27 \\
(0.81- \\
1.99) \\
0.297\end{array}$ & $\begin{array}{l}1.02 \\
(0.39- \\
2.64) \\
0.973\end{array}$ & $\begin{array}{l}1.18 \\
(0.81- \\
1.72), \\
0.384\end{array}$ & $\begin{array}{l}1.19 \\
(0.82- \\
1.72) \\
0.360\end{array}$ \\
\hline & LNM & $\left(\begin{array}{l}(-) / \\
+)\end{array}\right.$ & $\begin{array}{l}0.56(0.24- \\
1.32), 0.183\end{array}$ & $\begin{array}{l}1.02(0.71- \\
1.45), 0.924\end{array}$ & $\begin{array}{l}0.95 \\
(0.68- \\
1.35) \\
0.791\end{array}$ & $\begin{array}{l}0.56 \\
(0.24- \\
1.29) \\
0.172\end{array}$ & $\begin{array}{l}0.90 \\
(0.67- \\
1.20), \\
0.474\end{array}$ & $\begin{array}{l}0.90 \\
(0.68- \\
1.20), \\
0.473\end{array}$ \\
\hline & Stage & $\nabla-\nabla / \nabla-\square$ & $\begin{array}{l}0.33(0.10- \\
1.14), 0.079\end{array}$ & $\begin{array}{l}1.02(0.69- \\
1.50), 0.941\end{array}$ & $\begin{array}{l}0.92 \\
(0.63- \\
1.35) \\
0.685\end{array}$ & $\begin{array}{l}0.33 \\
(0.10- \\
1.12) \\
0.076\end{array}$ & $\begin{array}{l}0.85 \\
(0.61- \\
1.17), \\
0.317\end{array}$ & $\begin{array}{l}0.85 \\
(0.62- \\
1.16), \\
0.302\end{array}$ \\
\hline & ER & $(-) /$ & $\begin{array}{l}1.39(0.54- \\
3.58), 0.500\end{array}$ & $\begin{array}{l}1.02(0.69- \\
1.50), 0.933\end{array}$ & $\begin{array}{l}1.05 \\
(0.72- \\
1.53) \\
0.795\end{array}$ & $\begin{array}{l}1.38 \\
(0.54- \\
3.52) \\
0.503\end{array}$ & $\begin{array}{l}1.08 \\
(0.78- \\
1.48), \\
0.645\end{array}$ & $\begin{array}{l}1.07 \\
(0.78- \\
1.47) \\
0.660\end{array}$ \\
\hline & PR & $\left(\begin{array}{l}(-) / \\
+)\end{array}\right.$ & $\begin{array}{l}1.72(0.70- \\
4.26), 0.239\end{array}$ & $\begin{array}{l}1.11(0.77- \\
1.60), 0.572\end{array}$ & $\begin{array}{l}1.16 \\
(0.82- \\
1.65) \\
0.401\end{array}$ & $\begin{array}{l}1.66 \\
(0.68- \\
4.05) \\
0.269\end{array}$ & $\begin{array}{l}1.19 \\
(0.88- \\
1.60) \\
0.268\end{array}$ & $\begin{array}{l}1.17 \\
(0.88- \\
1.57), \\
0.286\end{array}$ \\
\hline & HER2 & $\left(\begin{array}{l}(-) / \\
+)\end{array}\right.$ & $\begin{array}{l}0.52(0.17- \\
1.62), 0.259\end{array}$ & $\begin{array}{l}0.78(0.47- \\
1.28), 0.325\end{array}$ & $\begin{array}{l}0.75 \\
(0.46- \\
1.21) \\
0.234\end{array}$ & $\begin{array}{l}0.57 \\
(0.19- \\
1.76) \\
0.330\end{array}$ & $\begin{array}{l}0.75 \\
(0.50- \\
1.13), \\
0.175\end{array}$ & $\begin{array}{l}0.78 \\
(0.53- \\
1.16), \\
0.215\end{array}$ \\
\hline
\end{tabular}

SNP, single nucleotide polymorphism; OR, odds ratio; $95 \% \mathrm{Cl}, 95 \%$ confidence interval; ER, estrogen receptor; RP, progesterone receptor; HER2, human epidermal growth factor receptor 2; LNM, lymph node metastasis.

$p$ values were calculated by logistic regression analysis with adjustments for age.

$p<0.05$ indicates statistical significance. 


$\begin{array}{llll}\text { Ki-67 } & < & 0.50(0.21- & 0.66(0.44- \\ & 10 \% / & 1.20), 0.119 & 1.01), 0.054 \\ & \geq & & \\ & 10 \% & & \end{array}$

$\begin{array}{llll}0.64 & 0.59 & 0.68 & 0.69 \\ (0.43- & (0.25- & (0.49- & (0.50- \\ 0.96), & 1.39), & 0.95), & 0.95) \\ 0.030 & 0.225 & \mathbf{0 . 0 2 4} & \mathbf{0 . 0 2 5}\end{array}$

SNP, single nucleotide polymorphism; OR, odds ratio; $95 \% \mathrm{Cl}, 95 \%$ confidence interval; ER, estrogen receptor; RP, progesterone receptor; HER2, human epidermal growth factor receptor 2; LNM, lymph node metastasis.

$p$ values were calculated by logistic regression analysis with adjustments for age.

$p<0.05$ indicates statistical significance.

Figure 1 represented the reconstructed LD plot, and had three blocks in LRRC3B SNPs (Block 1, rs112276562 and rs6790894; Block 2, rs6551121, rs6551122, rs1907168 and rs73150416; and Block 3, rs12635768, rs6551130, rs78205284 and rs6788033). Moreover, 'GATT' haplotype in the Block 2 had a higher risk for BC (OR $=1.29,95 \%$ Cl: $1.00-$ $1.65, p=0.048$, Supplementary Table 4).

According to GEPIA database, $\angle R R C 3 B$ gene was down-regulated in $\mathrm{BC}(p<0.01$, Supplementary Fig. 1$)$. Kaplan-Meier plotter showed high expression of $\angle R R C 3 B$ was related to better $\mathrm{BC}$ overall survival $(\mathrm{HR}=0.83,95 \% \mathrm{Cl}: 0.71-0.97, p=$ 0.017, Supplementary Fig. 2). We speculated that $\angle R R C 3 B$ is a tumor suppressor gene, but more convincing studies are needed to validate the conclusions because these clinical data are from the database.

\section{Discussion}

This study investigated the effect of $\angle R R C 3 B$ genetic variation on $\mathrm{BC}$ risk in a Chinese Han population. The result revealed that rs1907168 conferred to reduced BC risk overall. In additional, rs1907168 decreased the risk of BC while rs78205284 increased BC susceptibility among the population at age $\leq 51$ years. Noticeably, clinical parameters such as the status of PR and Ki67 and tumor size were significantly associated with $L R R C 3 B$ polymorphism. Furthermore, we found that the existence of three blocks in $\angle R R C 3 B$ SNPs and the association of 'GATT' haplotype with an increased BC risk. Our results suggested $\angle R R C 3 B$ variants might be susceptibility markers for $\mathrm{BC}$. Specially, this is firstly demonstrated the effects of $\angle R R C 3 B$ SNPs on BC susceptibility.

$\angle R R C 3 B$, located at 3p24, contained a leucine-rich repeat N-terminal domain (Leu, $>13 \%$ ). The protein-conserved region of LRRC3B exists CAMP- and CGMP-dependent protein kinase phosphorylation sites; thus, related to cell differentiation, cycle regulation, proliferation and invasion $[18,19]$. Several studies have revealed $\angle R R C 3 B$ could be as a tumor suppressor in carcinogenesis $[20,21]$. Formerly, the expression of $\angle R R C 3 B$ was reduced in $B C$ tissues and associated with tumor grade of $\mathrm{BC}[13]$. Consistently, we also found that $\angle R R C 3 B$ was down-regulated in $\mathrm{BC}$ and low expression of $L R R C 3 B$ was related to a poor overall survival for $\mathrm{BC}$ based on bioinformatics analysis. Moreover, $L R R C 3 B$ might serve a protective role in preventing bupivacaine-induced $\mathrm{BC}$ recurrence and metastasis[15]. These results hint us that $L R R C 3 B$ has an important role in $\mathrm{BC}$ occurrence and progression.

Genetic variations in $\angle R R C 3 B$ might be associate with the development and progress of disease. However, there is no finding of the related research about $\angle R R C 3 B$ polymorphisms. In this study, $L R R C 3 B$ rs $1907168(\mathrm{~A} / T)$ variant have a protective effect in BC risk. Further, stratified analysis showed that rs1907168 decreased while rs78205284 increased BC susceptibility among the population at age $\leq 51$ years. Notably, the peak age for BC is $40-50$ years in the Asian countries, whereas 60-70 years in the Western countries [22]. This finding suggested that age might affect the association of genetic variations and BCC, and that rs78205284 was a risk factor to BC among younger women in Chinese Han population. 
As $B C$ is a complex disease, the same variant might show a different response for BC risk across different tumor size, clinical stage and clinical features $[23,24]$. We further evaluated association of $L R R C 3 B$ polymorphisms and clinical features of BC, including tumor size, lymph node metastasis, clinical stage, the statuses of ER, PR, Her-2, and Ki67. We found that rs6551122 and rs12635768 polymorphisms were associated with a smaller BC tumor size $(<2 \mathrm{~cm})$. In addition, rs112276562, rs6551122 and rs73150416 variants contributed to a lower incidence of PR-positive BC, suggesting that $\angle R R C 3 B$ polymorphisms might involve in the pathogenesis of PR-positive BC. Moreover, rs6788033 was associated with a lower level of $\mathrm{Ki}-67$. Our findings showed $\angle R R C 3 B$ polymorphisms were associated with tumors size and PR / Ki-67 status, which might act as predictors for BC occurrence and development.

Our study provided the evidence that $\angle R R C 3 B$ variants were associated with $\mathrm{BC}$ susceptibility and related to clinical characteristic of $\mathrm{BC}$ patients. Inevitably, there were several intrinsic limitations in this study. First, data on $L R R C 3 B$ SNPS potential function, and $\angle R R C 3 B$ expression/survival in $\mathrm{BC}$ were predicted in silico analysis only; thus, further functional assay is necessary to explore the functions and underlying mechanisms of these polymorphisms. Second, gene-toenvironment interactions could not be further analyzed due to the unavailability of relevant information; therefore, additional studies will be required.

\section{Conclusion}

In conclusion, we firstly reported that $\angle R R C 3 B$ polymorphisms might contribute to individual susceptibility and progression to $\mathrm{BC}$ among Chinese Han females. The study might help to understand the possible effect of $L R R C 3 B$ variants in the development of $\mathrm{BC}$.

\section{Declarations}

\section{Ethics approval and consent to participate}

The study was approved by the Ethics Committee of Xizang Minzu University and in accordance with the 1964 Helsinki Declaration. Written informed consent was obtained.

\section{Consent for publication}

Not applicable.

\section{Availability of data and material}

All the data regarding the findings are available within the manuscript.

\section{Competing interests}

The authors declare that they have no conflict of interest.

\section{Funding}

No.

\section{Authors' contributions}

Tianbo Jin: Writing - original draft, Conceptualization. Linna Peng, Shishi Xing, and Dandan Li: Methodology. Chunjuan He and Xue He: Data curation. Li Wang: Conceptualization, Writing - review \& editing. 
We are grateful to the individuals who participated in this study.

\section{References}

1. Bray F, Ferlay J, Soerjomataram I, Siegel RL, Torre LA, Jemal A. Global cancer statistics 2018: GLOBOCAN estimates of incidence and mortality worldwide for 36 cancers in 185 countries. Cancer J Clin. 2018;68(6):394-424.

2. Chen W, Zheng R, Baade PD, Zhang S, Zeng H, Bray F, Jemal A, Yu XQ, He J. Cancer statistics in China, 2015. Cancer J Clin. 2016;66(2):115-32.

3. Sparano JA, Gray RJ, Makower DF, Pritchard KI, Albain KS, Hayes DF, Geyer CE Jr., Dees EC, Perez EA, Olson JA, Jr. et al: Prospective Validation of a 21-Gene Expression Assay in Breast Cancer. The New England journal of medicine 2015, 373(21):2005-2014.

4. Howell A, Anderson AS, Clarke RB, Duffy SW, Evans DG, Garcia-Closas M, Gescher AJ, Key TJ, Saxton JM, Harvie MN. Risk determination and prevention of breast cancer. Breast cancer research: BCR. 2014;16(5):446.

5. Xia P, Li B, Geng T, Deng Z, Dang C, Chang D, Kang L, Jin T, Chen C. FGFR2 gene polymorphisms are associated with breast cancer risk in the Han Chinese population. American journal of cancer research. 2015;5(5):1854-61.

6. Dai ZJ, Liu XH, Ma YF, Kang HF, Jin TB, Dai ZM, Guan HT, Wang M, Liu K, Dai C, et al. Association Between Single Nucleotide Polymorphisms in DNA Polymerase Kappa Gene and Breast Cancer Risk in Chinese Han Population: A STROBE-Compliant Observational Study. Medicine. 2016;95(2):e2466.

7. Ren HT, Li YM, Wang XJ, Kang HF, Jin TB, Ma XB, Liu XH, Wang M, Liu K, Xu P, et al. PD-1 rs2227982 Polymorphism Is Associated With the Decreased Risk of Breast Cancer in Northwest Chinese Women: A Hospital-Based Observational Study. Medicine. 2016;95(21):e3760.

8. Clark HF, Gurney AL, Abaya E, Baker K, Baldwin D, Brush J, Chen J, Chow B, Chui C, Crowley C, et al. The secreted protein discovery initiative (SPDI), a large-scale effort to identify novel human secreted and transmembrane proteins: a bioinformatics assessment. Genome research. 2003;13(10):2265-70.

9. Kobe B, Deisenhofer J. A structural basis of the interactions between leucine-rich repeats and protein ligands. Nature. 1995;374(6518):183-6.

10. Xu ZM, Gao WR, Mei Q, Chen J, Lu J. The novel gene LRP15 is regulated by DNA methylation and confers increased efficiency of DNA repair of ultraviolet-induced DNA damage. BMB Rep. 2008;41(3):230-5.

11. Guo Y, Zhang R, Shen S, Wei Y, Salama SM, Fleischer T, Bjaanaes MM, Karlsson A, Planck M, Su L, et al: DNA Methylation of LRRC3B: A Biomarker for Survival of Early-Stage Non-Small Cell Lung Cancer Patients. 2018, 27(12):1527-1535.

12. Kim M, Kim JH, Jang HR, Kim HM, Lee CW, Noh SM, Song KS, Cho JS, Jeong HY, Hahn Y, et al. LRRC3B, encoding a leucine-rich repeat-containing protein, is a putative tumor suppressor gene in gastric cancer. Cancer research. 2008;68(17):7147-55.

13. Wang Y, Peng Y, Zhou Y, Zhou Y, Zhong H. The clinical value of LRRC3B gene expression and promoter hypermethylation in breast carcinomas. Cell Biochem Biophys. 2014;70(2):1035-41.

14. Demokan S, Chuang AY, Pattani KM, Sidransky D, Koch W, Califano JA. Validation of nucleolar protein 4 as a novel methylated tumor suppressor gene in head and neck cancer. Oncol Rep. 2014;31(2):1014-20.

15. Li GS, Kong GY, Zou Y. Protective role of LRRC3B in preventing breast cancer metastasis and recurrence postbupivacaine. Oncology letters. 2017;14(4):5013-7.

16. Gabriel S, Ziaugra L, Tabbaa D. SNP genotyping using the Sequenom MassARRAY iPLEX platform. Current protocols in human genetics 2009, Chap. 2:Unit 2.12.. 
17. Thomas RK, Baker AC, Debiasi RM, Winckler W, Laframboise T, Lin WM, Wang M, Feng W, Zander T, MacConaill L, et al. High-throughput oncogene mutation profiling in human cancer. Nat Genet. 2007;39(3):347-51.

18. Bella J, Hindle KL, McEwan PA, Lovell SC. The leucine-rich repeat structure. Cell Mol Life Sci. 2008;65(15):2307-33.

19. Kan L, Li H, Zhang Y, Wang J, Niu H, Jiang H, Zhang M, Bai X, He P. LRRC3B is downregulated in non-small-cell lung cancer and inhibits cancer cell proliferation and invasion. Tumour biology: the journal of the International Society for Oncodevelopmental Biology Medicine. 2016;37(1):1113-20.

20. Haraldson K, Kashuba VI, Dmitriev AA, Senchenko VN, Kudryavtseva AV, Pavlova TV, Braga EA, Pronina IV, Kondratov $A G$, Rynditch AV, et al. LRRC3B gene is frequently epigenetically inactivated in several epithelial malignancies and inhibits cell growth and replication. Biochimie. 2012;94(5):1151-7.

21. Kondratov AG, Stoliar LA, Kvasha SM, Gordiyuk VV, Zgonnyk YM, Gerashchenko AV, Vozianov AF, Rynditch AV, Zabarovsky ER, Kashuba VI. Methylation pattern of the putative tumor-suppressor gene LRRC3B promoter in clear cell renal cell carcinomas. Mol Med Rep. 2012;5(2):509-12.

22. Leong SP, Shen ZZ, Liu TJ, Agarwal G, Tajima T, Paik NS, Sandelin K, Derossis A, Cody H, Foulkes WD. Is breast cancer the same disease in Asian and Western countries? World journal of surgery. 2010;34(10):2308-24.

23. Tian T, Wang M, Zheng Y, Yang T, Zhu W, Li H, Lin S, Liu K, Xu P, Deng Y, et al. Association of two FOXP3 polymorphisms with breast cancer susceptibility in Chinese Han women. Cancer management research. 2018;10:867-72.

24. Hirschhorn JN, Lohmueller K, Byrne E, Hirschhorn K. A comprehensive review of genetic association studies. Genetics in medicine: official journal of the American College of Medical Genetics. 2002;4(2):45-61.

\section{Figures}



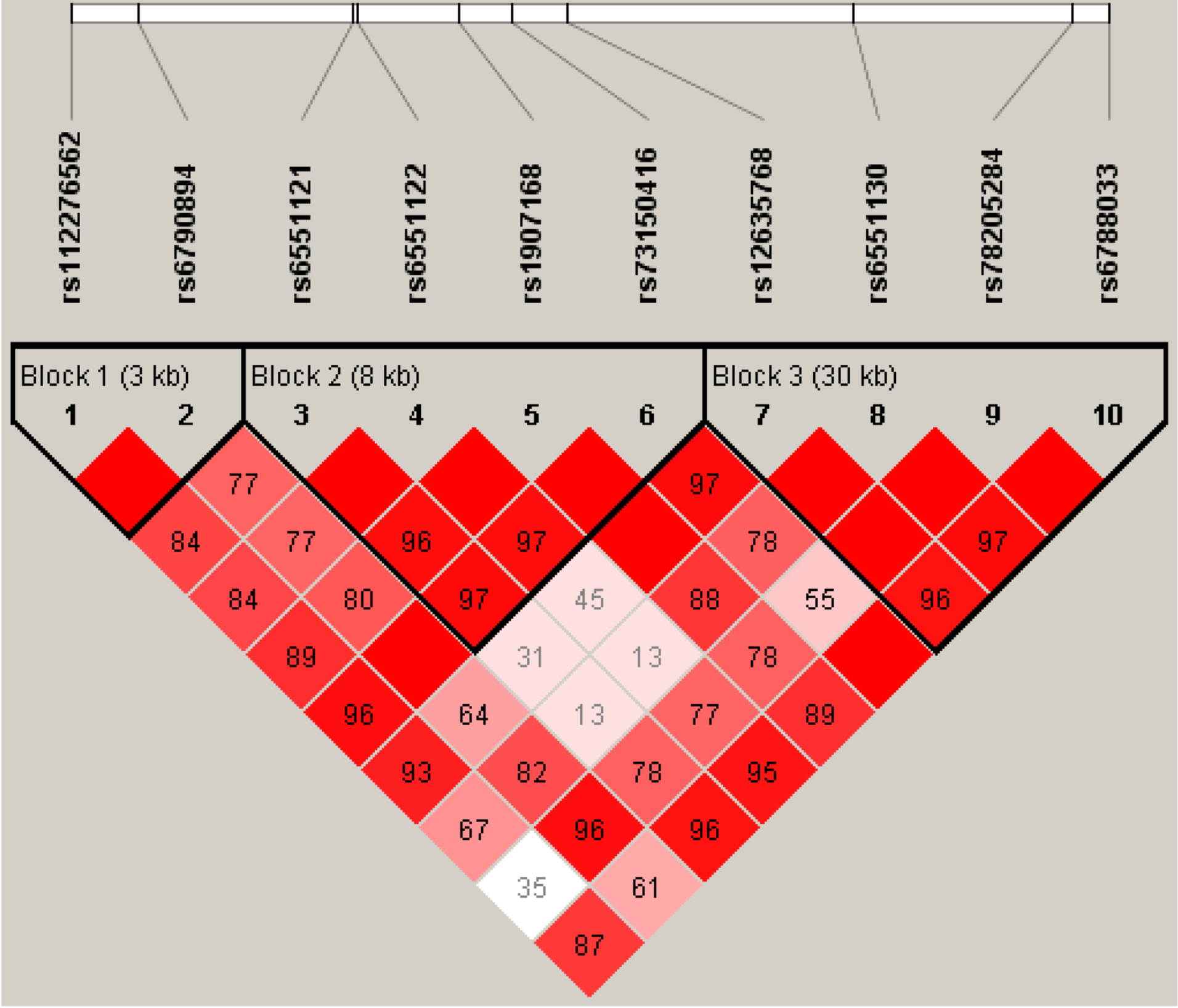

\section{Figure 1}

Haplotype block map for ten SNPs in the LRRC3B gene. The numbers inside the diamonds indicate the DQ for pairwise analyses.

\section{Supplementary Files}

This is a list of supplementary files associated with this preprint. Click to download.

- SupplementaryFigure1.tif

- SupplementaryFigure2.tif

- SupplementaryTable.docx 